\title{
A adoção de serviços cloud computing pelas empresas portuguesas: $O$ papel dos esforços de marketing
}

\author{
Nuno Fortes ${ }^{1}$, José Henrique Pereira² ${ }^{2}$ João Fontes da Costa ${ }^{3}$ \\ nuno.fortes@estgoh.ipc.pt, henrique_p6735@hotmail.com, fontesdacosta@fe.uc.pt \\ ${ }^{1}$ Instituto Politécnico de Coimbra, ESTGOH, Rua General Santos Costa, 3400-124 Oliveira do Hospital, Portugal \\ ${ }^{2}$ Instituto Politécnico de Coimbra, ESEC, Rua Dom João III - Solum, 3030-329 Coimbra, Portugal \\ ${ }^{3}$ Universidade de Coimbra, Faculdade de Economia, Avenida Dias da Silva, 165, 3004-512 Coimbra, Portugal
}

DOI: 10.17013/risti.18.33-48

\begin{abstract}
Resumo: Os serviços cloud computing são uma inovação tecnológica com vantagens evidentes para as organizações, embora a sua adoção pelas empresas portuguesas seja ainda incipiente. Os esforços de marketing podem desempenhar um papel relevante numa fase inicial de adoção destes serviços, enquanto potenciadores da intenção de utilização. A investigação na área é escassa e encontra-se numa fase embrionária. Este estudo é um contributo para o preenchimento desta lacuna, através da construção e validação empírica de um modelo que explica a forma como os esforços de marketing exercem a sua influência na intenção de utilização dos serviços cloud computing pelas empresas portuguesas. Os resultados obtidos no estudo confirmam o impacto positivo dos esforços de marketing na expectativa de esforço, condições facilitadoras, expectativa de performance e influência social, exercendo estas últimas três variáveis um impacto positivo na intenção de utilização de serviços cloud computing.
\end{abstract}

Palavras-chave: Cloud computing; Adoção de tecnologia; Esforços de marketing.

\section{The adoption of cloud computing services by Portuguese companies:} The impact of marketing efforts

\begin{abstract}
The cloud computing services are a technology innovation with an important set of benefits for organizations, although their adoption by Portuguese companies is still incipient. Marketing efforts can play an important role at an early stage of adoption of these services while enhancing the usage intention. The research in this field is scarce and is at an embryonic stage, which is a gap in the literature that must be overcome. This study seeks to be a contribution to filling this gap through the construction and empirical validation of a model that explains how marketing efforts exert their influence on the intention to use cloud computing services by Portuguese companies. The results of the study confirm the positive impact of marketing efforts on effort expectancy, facilitating conditions, performance expectancy and social influence, while the latter three variables exert a positive impact on the intention to use cloud computing services.
\end{abstract}

Keywords: Cloud computing; Technology acceptance; Marketing efforts. 


\section{Introdução}

Com a evolução da tecnologia surgiram novos modelos de negócio que têm vindo a moldar o mundo das Tecnologias de Informação (TI) (Sahoo, 2009). Armbrust et al. (2010) evidenciam o conceito cloud computing como sendo algo revolucionário nesta indústria. Este novo paradigma promete, essencialmente, a entrega de serviços de TI com as mesmas ou até mesmo novas funcionalidades das soluções já existentes, como também a redução de custos iniciais que impedem muitas empresas de implementar serviços tecnologicamente mais avançados (Marston, Li, Bandyopadhyay, Zhang, \& Ghalsasi, 2011). No modelo cloud computing, o software, a plataforma, ou a infraestrutura passam a ser fornecidos ao cliente como um serviço (Stuckenberg, Fielt, \& Loser, 2011).

Para sublinhar o potencial que os serviços cloud computing estão a alcançar, é necessário compreender de uma forma clara os determinantes e as diferentes questões envolvidas, tanto a nível do consumidor, como a nível do fornecedor (Marston et al., 2011). Do ponto de vista do consumidor, este começa a encontrar novas alternativas para reduzir custos em despesas com TI, acrescentando mais valor ao seu negócio e fazendo uma utilização mais rentável dos seus recursos e serviços TI (Böhm, Leimeister, Riedl, \& Krcmar 2011). Por outro lado, este novo serviço passa a ser visto como uma opção viável para substituir a infraestrutura atual de TI das empresas, o que faz com que os fornecedores de cloud computing se sintam pressionados a disponibilizar aos seus clientes novos modelos de software e novas alternativas de modelos de entrega de software (Francis, 2009).

Apesar deste novo paradigma apresentar um enorme potencial, cabe neste estudo verificar a realidade portuguesa, no que toca à adoção de serviços desta natureza. Um estudo realizado pela International Data Corporation (IDC) constatou que a adoção de serviços cloud computing pelas empresas portuguesas ainda é uma realidade um pouco distante (IDC Portugal, 2011). Este estudo debruçou-se sobre as 200 maiores organizações nacionais, tendo a IDC concluído que apenas 11\% já tinham implementado serviços privados de cloud computing, 7\% tinham a implementação em curso e $11 \%$ planeavam implementar no futuro. De realçar que 1/3 das empresas inquiridas referiu não tencionar adotar este tipo de soluções. No que respeita aos serviços públicos de cloud computing, o cenário é ainda menos encorajador. Apenas 4\% das organizações inquiridas já tinham aderido aos serviços cloud computing, enquanto $7 \%$ tinham em curso a contratação dos mesmos. A maioria das organizações inquiridas declarou não tencionar contratar este tipo de serviços, pelo menos para já. Num outro estudo da IDC sobre serviços cloud computing, os autores concluem que, apesar das enormes expectativas iniciais, as pequenas e médias empresas não têm adotado estes serviços tão rapidamente como previsto inicialmente e, como tal, são necessários esforços de marketing para potenciar a venda destes serviços àquelas empresas (IDC, 2007).

Segundo DeLone e McLean (1992), os trabalhos de investigação realizados no âmbito da utilização de sistemas de informação focam-se em três tipos de utilizadores: estudantes, profissionais das empresas e público em geral. Os utilizadores escolhidos para esta investigação foram profissionais de TI das empresas portuguesas, que tinham um papel de decisão na escolha de tecnologias a adotar pela empresa onde exercem a sua atividade. Para aqueles autores, o perfil demográfico, a experiência, a motivação e a atitude dos estudantes é bem diferente da dos profissionais das empresas no que toca a adotar uma nova tecnologia. 
Sendo os serviços cloud computing muito recentes em Portugal e escassa a literatura específica sobre a adoção destes serviços pelas empresas portuguesas, urge ultrapassar esta lacuna através da modelação dos determinantes da adoção desta tecnologia, auscultando os profissionais de TI responsáveis pela tomada de decisão nesta matéria. Por outro lado, é reconhecido que são necessários esforços de marketing para fomentar a adoção destes serviços pelas empresas (IDC, 2007). Deste modo, esta investigação procura compreender de que forma os esforços de marketing exercem o seu impacto na intenção de adoção de serviços cloud computing por parte das empresas portuguesas.

O presente trabalho tem a seguinte estrutura: após uma breve introdução ao tema, seguese uma revisão da literatura sobre o conceito e modelos de cloud computing. Na terceira secção apresenta-se o modelo de investigação, devidamente fundamentado. A quarta secção apresenta a metodologia seguida, bem como a sua justificação. Na quinta secção são analisados os resultados e na sexta e última secção são discutidos os resultados e apresentadas as conclusões.

\section{Revisão da literatura}

Apresenta-se, em seguida, a tecnologia em análise no presente estudo, a cloud computing, procurando explorar o conceito, as características, os modelos de infraestruturas e os modelos de entrega destes serviços.

\subsection{Conceito de cloud computing}

O conceito cloud computing tornou-se ao longo do tempo um termo comum e popular, ao qual está associada a utilização global de uma rede de computadores, que estão ligados a um número massivo de servidores físicos ou virtuais designados por "nuvem" (Hayes, 2008). Estes servidores podem ser utilizados por múltiplos utilizadores, sejam estes pessoas ou empresas. Neste panorama, o software é visto como um serviço que é prestado a um baixo custo e a uma grande quantidade de utilizadores (Prata, Fazendeiro, Augusto, Azevedo, \& Machado, 2013). Esta evolução consiste em ter vários cenários tecnológicos como hardware, virtualização, computação distribuída e serviços web e software disponíveis na internet, metaforicamente "na nuvem” (Sultan, 2010). As soluções cloud computing dão às empresas e utilizadores um fácil acesso ao potencial da computação (Wu, Lan, \& Lee, 2011). Ao colocarem todos os seus serviços na cloud, os utilizadores irão reduzir o seu custo total em TI (Marston et al, 2011). As soluções cloud computing oferecem, assim, benefícios económicos que as empresas não podem ignorar, pese embora sejam de considerar todos os problemas inerentes ao outsourcing de soluções de TI (Sáenz, Cámara, Calvo-Manzano, \& Arcilla, 2014).

O conceito de cloud computing émuitas das vezes utilizado para indicar um novo paradigma, que de uma forma flexível, disponibiliza recursos e serviços TI através da internet (Böhm et al., 2011). Vaquero et al. (2008) dão uma definição similar, argumentando que cloud computing baseia-se num conjunto de recursos virtualizados, facilmente utilizáveis e flexíveis. Esses recursos são dinamicamente reconfigurados a uma carga variável que permite a sua utilização ideal. Também Youseff, Butrico e Da Silva (2008) consideram cloud computing como sendo um novo paradigma de computação que permite que os 
seus consumidores utilizem temporariamente a infraestrutura de computação em rede fornecida como um serviço pelos respetivos fornecedores. Uma das definições de cloud computing mais amplamente utilizadas é a proposta pelo National Institute of Standards and Technology (NIST) elaborada por Mell e Grance (2011). Esta definição caracteriza o conceito como um modelo para permitir o acesso à rede de forma ubíqua, conveniente e sob procura a um conjunto compartilhado de recursos computacionais configuráveis que podem ser rapidamente fornecidos e substituídos com o mínimo esforço de gestão ou a mínima interação com o prestador de serviços (Mell e Grance, 2011).

\subsection{Características dos serviços cloud computing}

A Comissão Europeia no "Relatório do Grupo de Peritos" (Schubert, Jeffery, \& NeideckerLutz, 2010) resume as características mais comuns da computação em nuvem. Uma delas é a escalabilidade, que consiste na capacidade que a infraestrutura básica tem de se expandir, adaptando-se ao número de utilizadores e aos dados suportados nas aplicações (Zhang, Cheng, \& Boutaba, 2010).

O relatório também menciona a confiança, definida como a capacidade que o fornecedor de serviços cloud computing tem de evitar a ocorrência de falhas ou interrupções no funcionamento dos serviços. Esta confiança resulta da qualidade do serviço prestada pelo fornecedor.

Schubert et al. (2010) referem ainda a importância da agilidade e adaptabilidade dos serviços cloud computing, que não são mais do que a capacidade destes, automaticamente e em tempo real, reagirem e se adaptarem às necessidades dos utilizadores, desde o tipo ao tamanho dos recursos necessários.

Uma característica não menos importante referida pelos autores é a disponibilidade do serviço. Este deverá ter a capacidade de mascarar pequenas falhas que eventualmente possam surgir, de maneira a que o utilizador não se aperceba que elas ocorreram.

É importante destacar também as características económicas dos serviços cloud computing. Estes serviços vêm contribuir para uma redução de custos, e que o utilizador passa a pagar apenas pelos serviços que subscreve (Zhang et al., 2010). Como estes serviços não exigem por parte do utilizador um investimento em infraestruturas nas suas instalações, será possível responder de forma imediata às necessidades de alteração do sistema de TI do utilizador, sem que este tenha que esperar pela instalação de hardware. Resumindo, as soluções cloud computing passam a ser a melhor e mais rápida resposta de mercado. Para além da economia já referida, os serviços cloud computing devem garantir o retorno do investimento, na medida em que o esforço financeiro realizado deverá mais do que compensar os benefícios financeiros recolhidos. Acresce que, com a adoção de soluções de cloud computing, as despesas de capital em infraestruturas de TI transformam-se em despesas operacionais. No entanto, Schubert et al. (2010) referem que a relação custo/benefício real nem sempre é visível, pelo que será sempre necessário estudar qual a solução mais benéfica para cada empresa.

Os serviços cloud computing contribuem também, de acordo com Schubert et al. (2010), para um fácil ajuste do consumo energético e de emissões de carbono, reduzindo assim a pegada ecológica, nomeadamente o consumo de energia, devido ao melhor controlo e ajustamento automático do aumento ou redução dos recursos. 
Os referidos autores identificam também alguns aspetos tecnológicos que advêm da utilização de serviços cloud computing, começando pela virtualização, que descreve como sendo a camada de abstração tecnológica que esconde complexos sistemas técnicos do utilizador e lhe oferece flexibilidade adicional, proporcionando maior facilidade de utilização.

Destacam igualmente a possibilidade de vários utilizadores em diferentes locais acederem a bases de dados comuns, de forma segura, não estando dependentes de um único dispositivo. A segurança é vista pelos autores como um processo ou conjunto de etapas que ajudam a evitar que os dados sejam vistos ou acedidos por pessoas ou sistemas não autorizados. A privacidade dos dados pessoais, sendo um aspeto crítico do cloud computing, é muitas vezes vista como um aspeto da segurança. A conformidade traduz-se no cumprimento da legislação e regulamentação vigentes.

\subsection{Modelos de infraestrutura de cloud computing}

Existem quatro modelos de infraestrutura de cloud computing: cloud pública, cloud privada, cloud comunitária e cloud híbrida (Hogan \& Sokol, 2013; Zhang et al., 2010).

O modelo de cloud pública assenta numa disponibilidade quase imediata para qualquer utilizador, desde que detenha um acesso à internet, não lhe conferindo no entanto qualquer controlo sobre a mesma (Zhang et al., 2010). É visto como um modelo de implementação que é disponibilizado publicamente através do modelo pay-peruse, sendo que a infraestrutura de cloud é disponibilizada para uma utilização aberta ao público em geral. Pode ser gerida e operacionalizada por um setor empresarial, académico ou organização governamental.

Já o modelo de cloud privada, por norma está localizado na infraestrutura privada, nas instalações da organização, e sob o seu controlo, competindo à organização a escolha do modelo a implementar, analisados os prós e contras de cada alternativa (Zhang et al. 2010). Neste modelo, a infraestrutura cloud computing é disponibilizada para utilização exclusiva da organização proprietária.

O modelo de cloud comunitária refere-se a uma infraestrutura controlada e partilhada, em termos de dados e aplicações, por um conjunto de organizações com interesses comuns (Zhang et al., 2010). Tais interesses poderão residir, por exemplo, em requisitos específicos de segurança ou em missões de âmbito semelhante.

Por último, o modelo de cloud híbrida consiste na composição de diferentes modelos de cloud que interagem entre si (Hogan \& Sokol, 2013; Zhang et al., 2010). Neste modelo, os utilizadores são seletivos quanto à informação que disponibilizam na cloud pública, procurando normalmente manter os dados e o processamento da informação crítica de negócio na sua cloud privada, sob o seu controle.

\subsection{Modelos de entrega de serviços cloud computing}

Foram identificados três tipos de modelos de entrega de serviços cloud computing: o software como serviço (SaaS), a plataforma como serviço (PaaS) e a infraestrutura como Serviço (IaaS) (Vaquero et al., 2008; Zhang et al., 2010)).

O mais conhecido é o modelo SaaS, que ocorre quando as aplicações funcionam diretamente na cloud, sem existir a necessidade destas serem instaladas no computador 
do utilizador (Marston et al., 2011). Neste modelo, o cliente recebe do fornecedor apenas a aplicação, podendo partilhá-la com outros utilizadores, os quais podem aceder às aplicações de diferentes locais, onde tenham um dispositivo com acesso a internet (Vaquero et al., 2008).

No modelo PaaS, o fornecedor disponibiliza ao cliente uma plataforma de sistemas que permite que as aplicações dos clientes funcionem (Vaquero et al., 2008). Esta plataforma proporciona ao cliente um ambiente de interfaces de desenvolvimento de aplicações que interagem com outras armazenadas na cloud (Leimeister et al., 2010). Este modelo é normalmente utilizado por empresas que têm necessidade de subscrever serviços no imediato e a um baixo custo, com o objetivo de desenvolverem as suas aplicações e programas sobre uma plataforma.

Por último, o modelo IaaS disponibiliza ao cliente uma potencialidade computacional e capacidade de armazenamento necessárias para que o utilizador utilize os seus ficheiros e programas. Neste modelo, o fornecedor apenas disponibiliza a infraestrutura que o cliente necessita, podendo aumentá-la ou diminui-la mediante as suas necessidades (Vaquero et al., 2008).

\section{Modelo de investigação}

O modelo de investigação proposto neste trabalho tem como base a teoria unificada de aceitação e uso de tecnologia (UTAUT), desenvolvida por Venkatesh, Morris, Davis e Davis (2003), a qual foi reforçada com a inclusão dos esforços de marketing, indispensáveis na fase inicial de adoção deste tipo de serviços (Lin, Wang, \& Hwang, 2010).

\subsection{Teoria unificada de aceitação e uso de tecnologia}

A UTAUT surgiu com o objetivo de integrar a teoria fragmentada e a pesquisa sobre a aceitação individual das TI num único modelo teórico que integrasse os elementos essenciais de outros modelos/teorias (Venkatesh et al., 2003). Este modelo visa a explicação da aceitação e utilização da tecnologia em contexto organizacional.

De acordo com a UTAUT, a intenção e a utilização de tecnologia são determinadas por quatro variáveis chave: condições facilitadoras, expectativa de esforço, expectativa de performance e influência social. As condições facilitadoras são definidas como o grau em que um indivíduo acredita que existe uma infraestrutura organizacional e técnica para suportar a utilização do sistema. Por outro lado, a expectativa de performance refere-se ao grau em que um indivíduo acredita que a utilização do sistema o irá ajudar a obter ganhos de desempenho no trabalho. Já a expectativa de esforço define-se como o grau de facilidade associado à utilização do sistema. Por fim, a influência social é o grau em que um indivíduo percebe que os seus referentes sociais acreditam que ele deve usar o novo sistema.

A utilização de tecnologia é explicada diretamente pela intenção de utilização e pelas condições facilitadoras. Por seu turno, a intenção de utilização é determinada diretamente pela expectativa de desempenho, pela expectativa de esforço e pela influência social. Esta teoria integra ainda quatro variáveis moderadoras das relações entre as quatro variáveis 
nucleares e a intenção e utilização de tecnologia: género, idade, voluntariedade de uso e experiência. Venkatesh et al. (2003) reportam que a UTAUT explica 70\% da variância da intenção de uso. Numa versão mais recente deste modelo, designada por UTAUT2, as condições facilitadoras passam a ser encaradas como um dos determinantes da intenção de utilização (Venkatesh, Thong e Xu, 2012).

A UTAUT é um dos modelos mais utilizados em estudos sobre a adoção e utilização de tecnologia, os quais têm vindo a corroborar que as condições facilitadoras, a expectativa de esforço, a expectativa de performance e a influência social são determinantes da intenção de utilização de tecnologia em contexto organizacional (Im, Hong, \& Kang., 2011; Zhou, Lu, \& Wang, 2010), o que nos faz acreditar que estas relações também se verificarão no contexto de adoção de serviços cloud computing pelas empresas portuguesas. Deste modo, propõem-se as seguintes hipóteses:

H1: As condições facilitadoras têm um impacto positivo na intenção de utilização;

H2: A expectativa de esforço tem um impacto positivo na intenção de utilização;

H3: A expectativa de performance tem um impacto positivo na intenção de utilização.

H4: A influência social tem um impacto positivo na intenção de utilização.

\subsection{Esforços de marketing}

Os esforços de marketing definem-se como a perceção que os indivíduos têm do grau de esforço que os fornecedores de serviços cloud computing desenvolvem para que esta nova tecnologia seja percecionada como atrativa, eficiente, de baixo custo, fácil de adquirir e suficientemente conhecida para ser confiável (Wu, 2011). Estes esforços são particularmente importantes na fase inicial de adoção de uma tecnologia (Lopez-Nicolas et al., 2008), tal como sucede com os serviços cloud computing no mercado empresarial português.

Do ponto de vista do marketing, a atitude e a decisão de compra de um indivíduo podem ser afetadas pelo marketing-mix, através de diversos tipos de estímulos e atividades promocionais (Wu, 2011). De acordo com Lin et al. (2010) e Wu (2011), o marketing-mix exerce uma influência significativa sobre a utilidade percebida, a facilidade de utilização percebida e a segurança e confiança. As duas primeiras variáveis estão incorporadas, no âmbito da UTAUT, na expectativa de desempenho e na expectativa de esforço, respetivamente (Venkatesh et al., 2003). Por sua vez, a segurança e a confiança atuam como atenuadores da incerteza, aumentando o controlo do indivíduo sobre o seu comportamento (Zand, 1972). Ora, o controlo do comportamento percebido é um construto incorporado na noção de condições facilitadoras da UTAUT (Venkatesh et al., 2003).

Por outro lado, os estudos de Lopez-Nicolas et al. (2008) e Wu (2011) dão suporte empírico ao impacto da influência dos media na influência social. Sendo a utilização dos media uma das várias ferramentas do marketing-mix, podemos assumir que os esforços de marketing têm um impacto positivo na influência social.

Neste contexto, propõem-se as seguintes hipóteses:

$\mathrm{H}_{5}$ : Os esforços de marketing têm um impacto positivo nas condições facilitadoras; 
H6: Os esforços de marketing têm um impacto positivo na expectativa de esforço;

H7: Os esforços de marketing têm um impacto positivo na expectativa de performance;

H8: Os esforços de marketing têm um impacto positivo na influência social.

\subsection{Modelo}

O modelo de investigação resultante da agregação das hipóteses acima enunciadas encontra-se representado na figura 1.

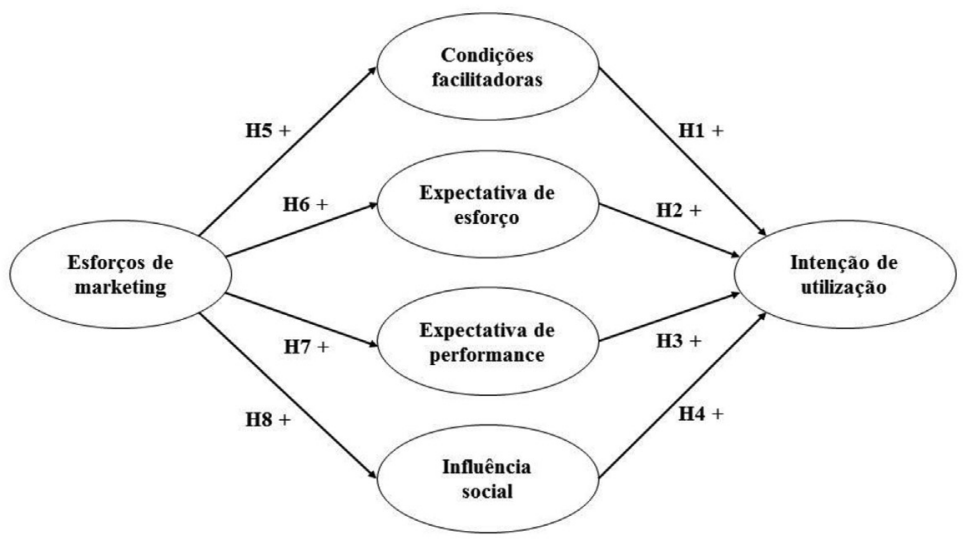

Figura 1 - Modelo de investigação.

\section{Metodologia}

De seguida é descrita a metodologia utilizada no estudo empírico. Começa-se por definir a população do estudo e por fazer uma caracterização da amostra. Posteriormente, apresenta-se o instrumento escolhido para a recolha de dados e, por fim, são detalhados os procedimentos adotados durante a recolha.

A população, isto é, o conjunto de elementos sobre os quais se vai debruçar a investigação, é constituída por todas as empresas portuguesas.

O estudo empírico teve por base uma amostra não-aleatória, de conveniência, constituída por 203 clientes empresariais da PT-Empresas, que são utilizadores de serviços cloud computing. Em cada empresa, foi inquirido o responsável pelo setor de TI. De acordo com a tabela 1, os inquiridos são maioritariamente do género masculino (88,2\%), têm idades entre os 26 e os 35 anos (38,9\%) e habilitações literárias ao nível da licenciatura (45,8\%). A maioria trabalha em empresas de média dimensão (34,0\%), da região Centro (43,3\%), do setor terciário $(56,7 \%)$, no departamento de TI $(56,7 \%)$ e são responsáveis de área $(41,9 \%)$. A maior parte dos inquiridos refere conhecer os serviços cloud computing há mais de 3 anos (38,9\%), mas utiliza-os há mais de um e há menos de dois anos (39,9\%). 


\begin{tabular}{|c|c|c|c|c|c|}
\hline & ariável & $\%$ & & Variável & $\%$ \\
\hline \multirow{2}{*}{ Género } & Masculino & 88,2 & \multirow{7}{*}{ Departamento } & Administração/Gerência & 20,2 \\
\hline & Feminino & 11,8 & & Comercial & 4,9 \\
\hline \multirow{4}{*}{ Idade } & $\leq 25$ anos & 4,4 & & Compras & 2,5 \\
\hline & 26-35 anos & 38,9 & & Financeiro & 8,4 \\
\hline & $36-45$ anos & 31,5 & & Recursos humanos & 1,5 \\
\hline & $>45$ anos & 25,1 & & Tec. de informação & 56,7 \\
\hline \multirow{6}{*}{ Região } & Norte & 34,0 & & Outro & 5,9 \\
\hline & Centro & 43,3 & \multirow{5}{*}{$\begin{array}{l}\text { Cargo ocupado } \\
\text { na organização }\end{array}$} & Administrador/Gerente & 23,2 \\
\hline & Lisboa & 20,2 & & Responsável de área & 41,9 \\
\hline & Alentejo & 0,5 & & Técnico & 11,8 \\
\hline & Algarve & 1,0 & & Administrativo & 21,7 \\
\hline & R. A. Dos Açores & 1,0 & & Outro & 1,5 \\
\hline \multirow{6}{*}{$\begin{array}{l}\text { Habilitações } \\
\text { literárias }\end{array}$} & $2^{\circ}$ ciclo ensino básico & 1,5 & \multirow{4}{*}{$\begin{array}{l}\text { Tempo de } \\
\text { conhecimento }\end{array}$} & $<1$ ano & 3 \\
\hline & $3^{\circ}$ ciclo ensino básico & 3,4 & & 1 a 2 anos & 24,1 \\
\hline & Ensino secundário & 22,2 & & 2 a 3 anos & 34 \\
\hline & Bacharelato & 4,9 & & + de 3 anos & 38,9 \\
\hline & Licenciatura & 45,8 & \multirow{4}{*}{$\begin{array}{l}\text { Tempo de } \\
\text { utilização }\end{array}$} & $<1$ ano & 29,1 \\
\hline & Mestrado & 22,2 & & 1 a 2 anos & 39,9 \\
\hline \multirow{5}{*}{$\begin{array}{l}\text { Dimensão da } \\
\text { empresa }\end{array}$} & Micro & 12,3 & & 2 a 3 anos & 19,7 \\
\hline & Pequena & 24,1 & & + de 3 anos & 11,3 \\
\hline & Média & 37,4 & \multirow{3}{*}{$\begin{array}{l}\text { Setor de } \\
\text { atividade }\end{array}$} & Setor primário & 7,4 \\
\hline & Grande & 26,1 & & Setor secundário & 35 \\
\hline & & & & Setor terciário & 57,6 \\
\hline
\end{tabular}

Tabela 1 - Caracterização da amostra.

O instrumento de recolha de dados adotado foi um questionário. Este foi dividido em duas partes principais: a primeira incidiu nas características sociodemográficas do inquirido e nas características da empresa onde trabalha e a segunda contemplou questões relativas à adoção dos serviços cloud computing. As escalas propostas para o questionário resultaram de uma revisão da literatura abrangente nas áreas do comportamento do consumidor, sistemas de informação e e-marketing e foram adaptadas a partir dos trabalhos de Venkatesh et al. (2003), para os construtos intenção de utilização (INT), condições facilitadoras (CF), expectativa de esforço (EE), expectativa de performance (EP) e influência social (IS), e de Wu (2011), para o construto esforços de marketing. Todos os construtos foram medidos através de escalas de Likert de 7 pontos (1 - discordo totalmente a 7 - concordo totalmente). Foi realizado um pré-teste ao questionário junto de uma amostra constituída por 30 empresas, com o objetivo de aferir a clareza das perguntas, a organização do questionário, o tempo de preenchimento e recolher 
sugestões de melhoria. Através de tratamento estatístico adequado, foi possível concluir que as escalas utilizadas eram unidimensionais e tinham adequada consistência interna.

A recolha de dados processou-se com recurso à plataforma Google Drive, onde foi alojado o questionário. A escolha desta ferramenta justificou-se por ser gratuita e pela facilidade de utilização e de armazenamento de dados. Os potenciais inquiridos foram contactados por e-mail, sendo convidados a responder ao questionário a partir de um link para a página de alojamento. Em alguns casos, os inquiridos foram contactados via telefone, por forma a aumentar a probabilidade de obtenção de respostas. O link de acesso ao questionário esteve ativo durante cerca de 6 meses, entre setembro de 2014 e fevereiro de 2015 .

\section{Resultados}

O tratamento estatístico dos dados foi realizado através da modelação de equações estruturais com a metodologia partial least squares (PLS-SEM). A utilização desta metodologia justifica-se, porque a distribuição dos dados é não normal e o objetivo do estudo é debruçar-se sobre as inter-relações complexas entre um conjunto alargado de fatores, as quais podem ser difíceis de captar utilizando a modelação de equações estruturais baseada nas covariações (Chin, 2010). Os resultados obtidos com a metodologia PLS-SEM foram estimados usando o software SmartPLS 2.0.

O modelo de medida foi avaliado em termos de fiabilidade, validade convergente e validade discriminante. A tabela 2 apresenta os loadings, os cross-loadings e os valores-t dos indicadores de medida. Os valores-t foram obtidos através de bootstraping com 5000 iterações, indicando que todos os loadings são estatisticamente significativos ao nível de 1\%. Todos os itens têm loadings acima do valor mínimo de 0,7 (Götz, Liehr-Gobbers, \& Krafft, 2010), com exceção do item IS1. Atendendo a que este loading fica relativamente próximo do ponto de corte e a escala não apresenta problemas de fiabilidade, decidimos manter o item na análise subsequente. Estes resultados dão suporte à fiabilidade dos indicadores de medida.

\begin{tabular}{lccccccc}
\hline Itens & Loadings & $\begin{array}{c}\text { Cross } \\
\text { Loadings }\end{array}$ & Valores-t & Itens & Loadings & $\begin{array}{c}\text { Cross } \\
\text { Loadings }\end{array}$ & Valores-t \\
\hline INT1 & 0,903 & {$[0,453 ; 0,563]$} & 48,418 & EP2 & 0,864 & {$[0,382 ; 0,739]$} & 37,350 \\
\hline INT2 & 0,942 & {$[0,427 ; 0,596]$} & 84,921 & EP3 & 0,916 & {$[0,592 ; 0,741]$} & 85,610 \\
\hline INT3 & 0,942 & {$[0,428 ; 0,557]$} & 89,048 & EP4 & 0,875 & {$[0,472 ; 0,796]$} & 38,640 \\
\hline CF1 & 0,785 & {$[0,562 ; 0,709]$} & 22,095 & IS1 & 0,655 & {$[0,376 ; 0,530]$} & 10,802 \\
\hline CF2 & 0,853 & {$[0,382 ; 0,780]$} & 32,729 & IS2 & 0,821 & {$[0,432 ; 0,709]$} & 31,789 \\
\hline CF3 & 0,854 & {$[0,392 ; 0,759]$} & 37,048 & IS3 & 0,849 & {$[0,578 ; 0,636]$} & 45,858 \\
\hline CF4 & 0,848 & {$[0,568 ; 0,770]$} & 43,904 & IS4 & 0,905 & {$[0,585 ; 0,751]$} & 67,153 \\
\hline EE1 & 0,761 & {$[0,419 ; 0,641]$} & 18,487 & EMKT1 & 0,787 & {$[0,431 ; 0,686]$} & 29,411 \\
\hline EE2 & 0,883 & {$[0,346 ; 0,795]$} & 51,591 & EMKT2 & 0,720 & {$[0,397 ; 0,610]$} & 16,086 \\
\hline EE3 & 0,877 & {$[0,389 ; 0,823]$} & 47,120 & EMKT3 & 0,867 & {$[0,464 ; 0,787]$} & 45,112 \\
\hline EE4 & 0,837 & {$[0,431 ; 0,755]$} & 27,701 & EMKT4 & 0,807 & {$[0,450 ; 0,635]$} & 21,468 \\
\hline EP1 & 0,782 & {$[0,504 ; 0,654]$} & 21,232 & & & & \\
\hline
\end{tabular}

Tabela 2 - Loadings e cross-loadings. 
A tabela 3 descreve a variância média extraída (AVE), a fiabilidade compósita (CR) e as correlações de cada variável latente. Os valores da CR são maiores do que o valor mínimo recomendado de 0,6 (Götz et al., 2010), variando entre 0,874 e 0,950, o que nos permite concluir que todos os construtos têm adequada consistência interna. Para além disso, a AVE de todos os construtos é maior do que o ponto de corte mínimo de o,5 (Götz et al., 2010), o que assegura a sua validade convergente. Finalmente, de acordo com o critério de Fornell-Larcker, a validade discriminante foi obtida para cada construto, dado que a raiz quadrada da sua AVE é maior do que o valor absoluto das correlações com os restantes construtos (Fornell \& Larcker, 1981). Para além disso, de acordo com a tabela 3, os cross-loadings são maiores que os loadings em cada item, o que reforça a validade discriminante (Götz et al., 2010).

\begin{tabular}{lcccccccc}
\hline & \multirow{2}{*}{ AVE } & CR & \multicolumn{7}{c}{ Correlações } \\
\cline { 4 - 8 } & & & INT & CF & EE & EP & IS & EMKT \\
\hline INT & 0,864 & 0,950 & 0,929 & & & & & \\
\hline CF & 0,758 & 0,902 & 0,575 & 0,871 & & & & \\
\hline EE & 0,767 & 0,906 & 0,469 & 0,850 & 0,876 & & & \\
\hline EP & 0,741 & 0,919 & 0,575 & 0,791 & 0,851 & 0,861 & & \\
\hline IS & 0,661 & 0,885 & 0,615 & 0,781 & 0,796 & 0,773 & 0,813 & \\
\hline EMKT & 0,725 & 0,874 & 0,547 & 0,836 & 0,802 & 0,778 & 0,767 & 0,852 \\
\hline
\end{tabular}

Tabela 3 - AVE, CR e correlações entre os construtos.

O modelo estrutural, exposto na figura 2, foi avaliado pelo sinal, magnitude e significância estatística dos parâmetros das relações estruturais, assim como pela variância explicada $\left(\mathrm{R}^{2}\right)$ das variáveis latentes endógenas (Götz et al., 2010). Com exceção da relação $\mathrm{EE} \rightarrow \mathrm{INT}$, todas as restantes têm parâmetros com sinal compatível (positivo) com o previsto no modelo de investigação e são estatisticamente significativas ao nível de 1\%, o que permite confirmar todas as hipóteses exceto H2. Os resultados indicam que a variação da intenção de utilização é explicada em 46,3\% pela variação das condições facilitadoras, expectativa de esforço, expectativa de performance e influência social. Por outro lado, os esforços de marketing explicam 69,9\%, 64,4\%, 60,6\% e 58,8\% da variação das condições facilitadoras, expectativa de esforço, expectativa de performance e influência social, respetivamente.

A tabela 5 mostra os efeitos diretos, indiretos e totais entre as variáveis do modelo. A intenção de utilização é influenciada diretamente pelas condições facilitadoras, expectativa de esforço, expectativa de performance e influência social, e indiretamente pelos esforços de marketing. Por seu turno, esta última variável influencia diretamente as condições facilitadoras, expetativa de esforço, expectativa de performance e influência social. Todos os efeitos diretos, indiretos e totais são estatisticamente significativos ao nível de $0,1 \%$. Os esforços de marketing exercem o efeito direto mais forte sobre as condições facilitadoras $(\beta=0,836)$, enquanto que é à influência social que cabe o maior efeito direto sobre a intenção de utilização $(\beta=0,452)$. O único efeito indireto 


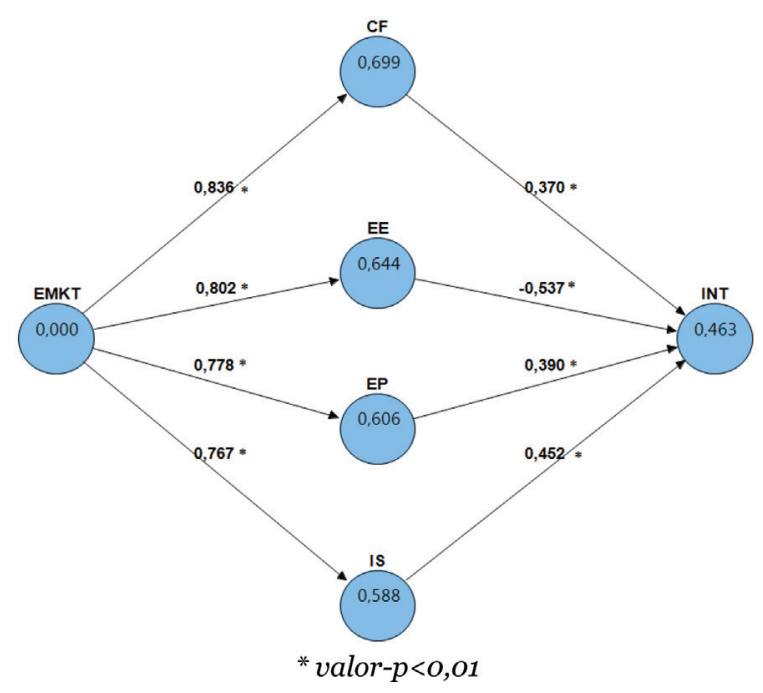

Figura 2 - Modelo estrutural

é exercido pelos esforços de marketing sobre a intenção de utilização $(\beta=0,529)$. Com exceção do efeito total dos esforços de marketing na intenção de utilização, que se deve exclusivamente ao efeito indireto, todos os restantes efeitos totais são coincidentes com os efeitos diretos. A importância dos esforços de marketing no modelo proposto é evidente, já que esta variável é a responsável pelo maior efeito total sobre a intenção de utilização $(\beta=0,529)$.

\begin{tabular}{|l|l|l|l|l|l|l|}
\hline \multirow{2}{*}{} & \multicolumn{2}{l}{ Efeitos diretos } & \multicolumn{2}{l|}{ Efeitos indiretos } & \multicolumn{2}{l|}{ Efeitos totais } \\
\cline { 2 - 7 } & Coeficientes & Valores-t & Coeficientes & Valores-t & Coeficientes & Valores-t \\
\hline $\mathrm{CF} \rightarrow \mathrm{INT}$ & 0,370 & 3,047 & & & 0,370 & 3,047 \\
\hline $\mathrm{EE} \rightarrow \mathrm{INT}$ & $-0,537$ & 3,359 & & & $-0,537$ & 3,359 \\
\hline $\mathrm{EP} \rightarrow \mathrm{INT}$ & 0,390 & 3,442 & & & 0,390 & 3,442 \\
\hline $\mathrm{IS} \rightarrow \mathrm{INT}$ & 0,452 & 3,877 & & & 0,452 & 3,877 \\
\hline EMKT $\rightarrow \mathrm{CF}$ & 0,836 & 26,353 & & & 0,836 & 26,353 \\
\hline EMKT $\rightarrow \mathrm{EE}$ & 0,802 & 25,226 & & & 0,802 & 25,226 \\
\hline EMKT $\rightarrow \mathrm{EP}$ & 0,778 & 25,598 & & & 0,778 & 25,598 \\
\hline EMKT $\rightarrow \mathrm{IS}$ & 0,767 & 19,168 & & & 0,767 & 19,168 \\
\hline EMKT $\rightarrow \mathrm{INT}$ & & & 0,529 & 10,994 & 0,529 & 10,994 \\
\hline
\end{tabular}

Tabela 5 - Efeitos diretos, indiretos e totais

\section{Discussão dos resultados e conclusões}

Os serviços cloud computing constituem uma inovação tecnológica que congrega um importante conjunto de vantagens decorrentes da sua utilização pelas empresas. Apesar 
disto, a adoção destes serviços em contexto organizacional é ainda bastante incipiente, em particular em Portugal. Reconhece-se que os esforços de marketing podem ser fundamentais para fomentar a adoção destes serviços.

A investigação produzida sobre a adoção e utilização de serviços cloud computing é escassa e encontra-se numa fase embrionária, o que constitui uma lacuna da literatura especializada que importa ultrapassar. Este estudo procura ser um contributo para o preenchimento dessa lacuna, através da construção e validação de um modelo que explica a forma como os esforços de marketing exercem o seu impacto na intenção de utilização dos serviços cloud computing pelas empresas.

O modelo proposto incorpora quatro constructos da UTAUT - intenção de utilização, condições facilitadoras, expectativa de performance, expectativa de esforço e influência social - os quais são complementados com a inclusão dos esforços de marketing.

O estudo empírico foi realizado através da aplicação de um questionário a uma amostra de 203 empresas portuguesas que utilizam serviços cloud computing.

Os resultados obtidos a partir da utilização da metodologia PLS-SEM confirmaram a fiabilidade, validade convergente e validade discriminante do modelo de medida. As hipóteses constantes do modelo estrutural foram integralmente validadas, com exceção da relação entre a expectativa de esforço e a intenção de utilização.

Os resultados indicam que quanto melhor for a perceção dos esforços de marketing desenvolvidos pelos fornecedores de serviços cloud computing, maior será a expectativa de performance e a expectativa de esforço, tal como defendido por Lin et al. (2010) e Wu (2011) ao referirem-se à utilidade percebida eà facilidade de utilização percebida. Recordese que, no âmbito do modelo UTAUT estas duas variáveis integram, respetivamente, a expectativa de performance e a expectativa de esforço (Venkatesh et al., 2003). Por outro lado, os resultados obtidos apontam para o impacto significativo dos esforços de marketing nas condições facilitadoras. Isto acontece porque os esforços de marketing, na medida em que fomentam as perceções de segurança e confiança, atuam como atenuadores da incerteza, aumentando o controlo comportamental percebido (Zand, 1972), que é um construto incorporado na noção de condições facilitadoras da UTAUT (Venkatesh et al., 2003). Por último, os resultados confirmam a influência significativa dos esforços de marketing na influência social, indo ao encontro dos estudos de LopezNicolas et al. (2008) e Wu (2011), que dão suporte empírico ao impacto da influência dos media, enquanto suporte de comunicação de marketing, na influência social.

Os resultados do modelo estrutural sustentam ainda o efeito significativo das condições facilitadoras, expectativa de performance e influência social na intenção de utilização de serviços cloud computing. Estes resultados vêm, assim, reforçar as conclusões da UTAUT (Venkatesh et al., 2003) e da UTAUT2 (Venkatesh et al., 2012), assim como dos trabalhos de Im et al. (2011), Lin e Anol (2008) e Zhou et al. (2010). Deste modo, podemos afirmar que quanto maior for a perceção dos responsáveis de TI das empresas portuguesas sobre as condições facilitadoras, a expectativa de performance e a influência social referentes aos serviços cloud computing, maior será a sua intenção de utilização destes serviços em contexto organizacional. Ao contrário do previsto no modelo UTAUT (Venkatesh et al., 2003), a expectativa de esforço evidenciou um impacto negativo na 
intenção de utilização dos serviços cloud computing, o que indicia que quanto mais difícil for utilizar estes serviços, maior é a probabilidade de virem a ser utilizados pelas empresas portuguesas. Este resultado parece sugerir que a perceção da facilidade de utilização induz aos profissionais de TI das empresas portuguesas sensações de falta de robustez do serviço prestado, que mitigam a probabilidade da sua utilização.

Deste estudo emergem relevantes contributos académicos e para o mundo empresarial. Em termos académicos, o principal contributo é a construção e validação de um modelo inovador que explica o papel decisivo que os esforços de marketing têm na intenção de utilizar serviços cloud computing pelas empresas. Este modelo integra no quadro da UTAUT os esforços de marketing enquanto variável preditora das condições facilitadoras, expectativa de performance, expectativa de esforço e influência social, exercendo por intermédio destas variáveis uma influência indireta, mas significativa, na intenção de utilização. Estamos, assim, perante uma abordagem inovadora no âmbito do modelo UTAUT, mas que reforça a grande maioria das conclusões desta teoria.

Para o mundo empresarial, esta investigação fornece contributos importantes ao dar indicações muito claras sobre os principais fatores que aumentam a probabilidade de utilização dos serviços cloud computing pelas empresas portuguesas. Em concreto, os fornecedores destes serviços deverão apostar na melhoria das respetivas condições facilitadoras, elevar as expectativas de performance dos utilizadores, aumentar a notoriedade social destas soluções tecnológicas e, a montante, apostar no reforço dos esforços de marketing direcionados para o mercado empresarial. A partir destas indicações, as empresas fornecedoras deste tipo de serviços poderão, de uma forma mais eficiente, agregar valor às soluções tecnológicas que comercializam e delinear estratégias de divulgação que deem resposta às exigências dos profissionais de TI aqui identificadas.

Tal como sucede com a maioria das investigações, este estudo apresenta algumas limitações que podem ser vistas como indicadores de sugestões para futuras investigações. A principal limitação encontra-se na dimensão da amostra e na base de recrutamento de respondentes, unicamente clientes da PT Empresas. Deste modo, recomenda-se a realização de um novo estudo empírico junto de uma amostra mais diversificada e de maior dimensão. Por outro lado, o modelo de investigação proposto mede apenas o impacto de um conjunto de variáveis na intenção de utilização. Sugerese, assim, a realização de um estudo longitudinal, que possa incluir o comportamento efetivo de utilização dos serviços cloud computing. Um estudo desta natureza permitiria aferir o poder preditivo da intenção de utilização sobre a utilização propriamente dita. Seria, igualmente, interessante a inclusão de outras variáveis preditoras da intenção e do comportamento, designadamente a segurança, a confiança e a privacidade.

\section{Referências}

Armbrust, M., Fox, A., Griffith, R., Joseph, A. D., Katz, R., Konwinski, A., ... \& Zaharia, M. (2010). A view of cloud computing. Communications of the ACM, 53(4), 50-58. doi:10.1145/1721654.1721672.

Böhm, M., Leimeister, S., Riedl, C., \& Krcmar, H. (2011). Cloud Computing-Outsourcing 2.0 or a new Business Model for IT Provisioning?. In Application management (pp. 31-56). Gabler. doi:10.1007/978-3-8349-6492-2_2. 
Chin, W. W. (2010). How to write up and report PLS analyses. In V. E. Vinzi, W. Chin, J. Henseler; H. Wang (Eds.), Handbook of Partial Least Squares: Concepts, Methods and Applications. Berlin: Springer.

DeLone, W. H., \& McLean, E. R. (1992). Information systems success: The quest for the dependent variable. Information Systems Research, 3(1), 60-95. doi:10477047/92/0301/0060.

Francis, L. (2009). Cloud Computing: Implications for Enterprise Software Vendors (ESV), System Design and Management Program. Thesis (S.M.) Massachusetts Institute of Technology.

Götz, O.; Liehr-Gobbers, K.; Krafft, M. (2010). Evaluation of structural equation models using the partial least squares (PLS) approach, Chapter 29 in V. E. Vinzi, W. Chin, J. Henseler; H. Wang (Eds.), Handbook of partial least squares: concepts, methods and applications. Berlin: Springer.

Hayes, B. (2008). Cloud Computing. Communications of the ACM, 51 (7), 9-11. doi:10.1145/1364782.1364786.

Hogan, M. \& Sokol, A. (2013). NIST Cloud Computing Standards Roadmap. NIST Special Publication, 500-291, Version 2.

IDC (2007). The Adoption of Software as a Service in Small and Medium-Sized Businesses: Perception Versus Reality, IDC.

IDC Portugal (2011). IT Service Management, Automation \& Cloud Computing. IDC.

Im, I., Hong, S., \& Kang, M. S. (2011). An international comparison of technology adoption: Testing the UTAUT model. Information \& Management, 48(1), 1-8. doi:10.1016/j.im.2010.09.001.

Leimeister S, Riedl, K., \& Krcmar, H. (2010). The Business Perspectives of Cloud Computing: Actors, Roles and Value Networks, ECIS 2010 Proceedings.

Lin, W. B., Wang, M. K., \& Hwang, K. P. (2010). The combined model of influencing on-line consumer behavior. Expert Systems with Applications, 37(4), 3236-3247. doi:10.1016/j.eswa.2009.09.056.

Marston, S., Li, Z., Bandyopadhyay, S., Zhang, J., \& Ghalsasi, A. (2011). Cloud computing-The business perspective. Decision Support Systems, 51(1), 176-189. doi:10.1016/j.dss.2010.12.006.

Mell, P., \& Grance, T. (2011). The NIST Definition of Cloud Computing. NIST special publication, 800 (145). doi:10.6028/NIST.SP.800-145.

Prata, P., Fazendeiro, P., Augusto, C., Azevedo, S., \& Machado, V. C. (2013). Ambiente Colaborativo para Avaliação de Cadeias de Abastecimento. RISTI - Revista Ibérica de Sistemas e Tecnologias de Informação, (12), 1-15. doi:10.4304/risti.12.1-15.

Sáenz, J., Cámara, M. D. L., Calvo-Manzano, J. A., \& Arcilla, M. (2014). Necesitan los proveedores de outsourcing una metodología para la provisión de servicios?. RISTI-Revista Ibérica de Sistemas e Tecnologias de Informação, (SPE1), 61-75. doi:10.4304/risti.e1.61-75 . 
Sahoo, M. (2009). IT Innovations: Evaluate, strategize, and invest. IT professional, 11(6), 16-22. doi:0.1109/MITP.2009.128.

Schubert, L., Jeffery, K., \& Neidecker-Lutz, B. (2010). The Future Of Cloud Computing, Opportunities for European.Cloud Computing Beyond. European Commission Informarion and Society Theme - Expert Group Report.

Stuckenberg, S., Fielt, E., \& Loser, T. (2011). The impact of software-as-a-service on business models of leading software vendors: experiences from three exploratory case studies. In Proceedings of the 15th Pacific Asia Conference on Information Systems (PACIS 2011). Queensland University of Technology.

Sultan, N. (2010). Cloud computing for education: A new dawn?. International Journal of Information Management, 30(2), 109-116. doi:10.1016/j.ijinfomgt.2009.09.004.

Vaquero, L. M., Rodero-Merino, L., Caceres, J., \& Lindner, M. (2008). A break in the clouds: towards a cloud definition. ACM SIGCOMM Computer Communication Review, 39(1), 50-55. doi:10.1145/1496091.1496100.

Venkatesh, V., Morris, M. G., Davis, G. B., \& Davis, F. D. (2003). User acceptance of information technology: Toward a unified view. MIS Quarterly, 27(3), 425-478. Retrieved from http://www.jstor.org/stable/30036540.

Venkatesh, V., Thong, J., \& Xu, X. (2012). Consumer acceptance and use of information technology: extending the unified theory of acceptance and use of technology. MIS Quarterly, 36(1), 157-178.

Wu, W. W. (2011). Developing an explorative model for SaaS adoption. Expert systems with applications, 38(12), 15057-15064. doi:10.1016/j.eswa.2011.05.039.

Wu, W. W., Lan, L. W., \& Lee, Y. T. (2011). Exploring decisive factors affecting an organization's SaaS adoption: A case study. International Journal of Information Management, 31(6), 556-563. doi:10.1016/j.ijinfomgt.2011.02.007.

Youseff, L., Butrico, M., \& Da Silva, D. (2008). Toward a unified ontology of cloud computing. In Grid Computing Environments Workshop, 2008. GCE'o8 (pp. 1-10). IEEE. doi:10.1109/GCE.2008.4738443.

Zand, D. E. (1972). Trust and managerial problem solving. Administrative science quarterly, 229-239. doi:10.2307/2393957.

Zhou, T., Lu, Y., \& Wang, B. (2010). Integrating TTF and UTAUT to explain mobile banking user adoption. Computers in Human Behavior, 26(4), 760-767. doi:10.1016/j.chb.2010.01.013. 